\title{
Uso educativo de la radio en tiempos de pandemia en escuelas rurales chilenas
}

Educational use of radio in times of pandemic in Chilean rural schools

Uso educacional do rádio em tempos de pandemia em escolas rurais chilenas

\section{Fernando GARIAS PÉREZ}

Universidad de Huelva, España / fercarias@gmail.com

\section{Ángel HERNANDO GÓMEZ}

Universidad de Huelva, España / angel.hernando@dpsi.uhu.es

Isidro MARÍN-GUTIÉRREZ

Universidad de Sevilla / imgutierrez@us.es

Chasqui. Revista Latinoamericana de Comunicación

N.o 146, abril-julio 2021 (Sección Monográfico, pp. 59.76)

ISSN 1390-1079 / e-ISSN 1390-924X

Ecuador: CIESPAL

Recibido: 07-12-2020/Aprobado: 22-02-2021 


\title{
Resumen
}

Se realizó una investigación de cómo se ha incorporado la radio educativa a los tiempos de pandemia, desde la experiencia de docentes en escuelas rurales del sur de Chile. El análisis se centra en cómo ha sido utilizada la radio como recurso pedagógico. Se utilizó una metodología cualitativa para el levantamiento de los datos, a partir de entrevistas al profesorado de escuelas. Los resultados demostraron prácticas innovadoras en los docentes para la producción de material didáctico en cuanto al uso de la radio, adaptando los contenidos al formato radiofónico y el contexto intercultural mapuche huilliche. Como conclusión se evidencia que la radio sigue siendo una alternativa ante las brechas de acceso a Internet.

Palabras clave: COVID-19; Chile; escuelas; radio; interculturalidad

\begin{abstract}
We will explore the ways in which educational radio has been incorporated into times of pandemic, based on the experience of teachers in rural schools in southern Chile. The analysis focuses on how radio has been used as a pedagogical resource based on the implementation of methodologies associated with it. A qualitative perspective was used to collect the data, from interviews via Meet with school teachers. The results showed innovative practices of the teaching staff for the production of didactic material based on the use of the radio, adapting the contents to the radio format and the intercultural mapuche huilliche context. Radio continues to be an alternative to Internet access gaps.
\end{abstract}

Key words: COVID-19; Chile; schools; radio; interculturality

\section{Resumo}

Exploraremos as maneiras como o rádio educacional foi incorporado em tempos de pandemia, com base na experiência dos professores em escolas rurais no sul do Chile. A análise centra-se na forma como o rádio tem sido utilizado como recurso pedagógico a partir da implementação das metodologias a ele associadas. Para a coleta de dados, utilizou-se uma perspectiva qualitativa, a partir de entrevistas por meio do Meet com professores da escola. Os resultados mostraram práticas inovadoras do corpo docente para a produção de material didático a partir do uso do rádio, adequando os conteúdos ao formato do rádio e ao contexto intercultural mapuche huilliche. $\mathrm{O}$ rádio continua sendo uma alternativa às lacunas de acesso à Internet.

Palavras-chave: COVID-19; Chile; escolas; rádio; interculturalidade 


\section{Introducción}

La radio como recurso educativo se ha constituido históricamente como un elemento para mediar los procesos de enseñanza-aprendizaje (Muñoz, 1994). Se ha visto soslayada por el advenimiento de otros tipos de medios de comunicación, pero ha sabido sortear los avances tecnológicos y mantenerse como una alternativa vigente en el campo de la educación hasta la actualidad (Jaimes, 2020).

Desde una perspectiva histórico-pedagógica, las propuestas radiofónicas han transversalizado los currículos y planes de estudio, fortaleciéndolos y dinamizándolos (Peppino, 1999). La radio genera instancias para el aprendizaje significativo, elevando la creatividad, la imaginación, el trabajo cooperativo e incluso el entretenimiento. La radio constituye uno de los mejores medios educativos, ya que impacta desde el sonido, desde los efectos e incluso desde el silencio (Méndiz Rojas, 2004). Es una fuente estimuladora para el aprendizaje como afirma Marshall McLuhan (1996): "la radio afecta a la gente de una forma muy íntima, de tú a tú, y ofrece todo un mundo de comunicación silenciosa entre el escritor, locutor y oyente. Este es el aspecto inmediato de la radio. Una experiencia íntima" (p.307).

A partir de estas aserciones y situándonos en el contexto del año 2020, donde una pandemia de carácter mundial por coronavirus ha transformado las relaciones entre las personas, prescindiendo del contacto directo e influyendo en las dinámicas de convivencia social, incluida la escuela como espacio de coexistencia, la radio surge y vuelve como una alternativa para suplir la modalidad histórica de presencialidad que determina a los procesos formativos. Emisoras de radios públicas, privadas, comunitarias y escolares, en diferentes modalidades (analógica y digital), han puesto al servicio de las instituciones educativas recursos técnicos y de transmisión para hacer llegar a sus usuarios, contenidos vinculados a programas y planes de estudio en todos los niveles formativos. Esto a partir de diferentes formatos radiofónicos como: Cápsulas educativas, podcast, radio clases o documentales breves (Cohen y Pereira, 2010). La radio tiene la ventaja de ser un medio de comunicación, que generalmente es de largo alcance, abarcando a muy bajo costo grandes extensiones de kilómetros, ofreciendo simultaneidad, instantaneidad y portabilidad (Jaimes, 2014), lo que la hace afectiva para fines educativos.

Desde este contexto es así como la radio se pone al servicio de la educación a distancia en tanto alternativa para lograr que los estudiantes que habitan en zonas rurales del sur de Chile, carentes de conexión y acceso a Internet en la coyuntura de la pandemia. Para que puedan recibir contenidos vinculados con los programas de estudio de sus respectivos niveles formativos, a través de cápsulas radiofónicas insertas en un espacio radial con frecuencia diaria de lunes a viernes llamado "A las orillas del Kutralhue". Este se transmite por Radio La Voz de la Costa y tiene una duración aproximada de 30 minutos. Además, 
se eleva el trabajo del profesorado, quienes en una labor sin precedentes, han adherido a sus competencias habilidades comunicacionales, mediáticas y digitales (Aguaded, Pérez, Peñalva, 2018), para poder producir objetos de aprendizaje.

\section{Marco Teórico}

\section{La radio y su rol educativo}

La radio como medio de comunicación social se ha vinculado con la educación a través de aquellas instituciones educativas que entre sus propuestas formativas contemplan el uso de medios como parte de los procesos de enseñanza y aprendizaje. Siendo una característica habitual transversalizar los currículos y planes de estudio, fortaleciéndolos y abordándolos desde una perspectiva más dinámica e innovadora, favoreciendo habilidades cognitivas, creativas, comunicativas y socio afectivas (Méndiz Rojas, 2004).

Como lo afirma Peppino (1999), en tanto sus contenidos, la radio educativa se plantea desde tres perspectivas; los formales, no formales e informales. Los primeros se vinculan con los programas nacionales de educación y dan respuesta a las exigencias de los currículos de manera rigurosa, es decir, se generan a partir de pautas preestablecidas y poseen una lógica didáctica para el abordaje de ciertas temáticas y áreas del conocimiento. En segundo término, los contenidos no formales son aquellos que refuerzan los aprendizajes, contemplando tópicos que, aunque no se declaran en los planes ni programas de estudio, pueden redundar positivamente en la formación del alumnado. Requieren cierta lógica en su planteamiento y deben dialogar con los temas centrales del currículo. En tanto los contenidos informales, son aquellos que son generados fuera de la lógica de los currículos y las estructuras rígidas del sistema educativo, aunque cumplen una función pedagógica de manera no deliberada, siendo que pueden ser asistidos por diferentes tipos de usuarios, independientemente del origen cultural y contexto geográfico en el que se encuentren. Son reutilizables y no se subscriben a una estructura rígida en tanto su producción, pues son de creación colectiva o responden a las necesidades de grupos diversos. Su pertinencia es histórica y en la actualidad han tomado un nuevo aire con la llegada de la radio digital e Internet, elementos que han potenciado su cobertura.

\section{Quédate en casa, estudia y escucha la radio}

Ante la situación pandémica que imposibilita asistir presencialmente a clases, la mayoría de las instituciones educativas a nivel mundial han optado por diversas modalidades de trabajo para darle continuidad a sus programas formativos. Según la Organización de las Naciones Unidas para la Educación, la Ciencia y la Cultura UNESCO, para mayo de 2020 más 1.200 millones de estudiantes de todos 
los niveles de enseñanza, habían dejado de recibir clases presenciales, de ellos 160 millones ubicados en Latinoamérica y el Caribe. Los países de la región y sus sistemas educativos han desarrollado propuestas vinculadas a la educación a distancia, utilizando diversos formatos y plataformas para el trabajo remoto, entre estos se encuentra la radio como recurso educativo.

Declara igualmente la UNESCO (2020) que, entre las modalidades de aprendizaje a distancia en línea, sobresale el uso de plataformas virtuales de aprendizaje asincrónico, utilizadas en 18 países. Mientras que, entre las formas de aprendizaje a distancia fuera de línea, 23 países realizan transmisiones de programas educativos por medios de comunicación tradicionales como la radio o la televisión.

Subyace un tema importante dentro de este contexto y es lo relacionado a las brechas en el acceso desigual a conexiones a Internet. Si bien Chile no aparece en las estadísticas como un país que porcentualmente presenta este problema, existe zonas, sobre todo rurales, donde la población tiene mayores dificultades económicas y sociales que no le permiten acceder a servicios y conexión a Internet. Esto dificulta la prosecución de estudios de quienes carecen de acceso oportuno a ciertas tecnologías digitales, quedando la radio como una alternativa para darle continuidad a los procesos formativos en contexto de confinamiento. Un caso es la provincia de Osorno al sur de Chile, donde la mayoría de los colegios y escuelas han tenido que implementar sistemas de educación remota que les permita continuar con los planes de estudio, siendo la radio una opción en aquellos lugares donde por razones económicas, sociales y de infraestructura no llega conexión a Internet.

\section{Dos formatos para la radio educativa hoy}

Existe diversidad de formatos para hacer radio educativa. Creemos pertinente centrarnos en dos tipos que representan las producciones realizadas por el profesorado que participó aportando información para este estudio.

Desde la perspectiva de la producción radiofónica y el impacto y eficacia del mensaje, los formatos se han redefinido y se caracterizan hoy por ser breves. Los contenidos deben ser precisos y concisos. Los tiempos deben ser acotados y resguardar una estructura que permita de manera inmediata la comprensión de la información. La producción de contenidos para uso educativo debería resguardar en tanto sus formatos, premisas que contemplen las características antes mencionadas (Muñoz, 1994). La cápsula radial y el podcast educativo dan cuenta de ello.

La cápsula radial es un micro formato radiofónico que no debe exceder los cinco minutos de duración. Se caracteriza por "comprimir" la información relevante en un mínimo de tiempo, enfocándose de manera precisa en la temática que se aborda. Puede presentarse de manera unitaria o en serie, siendo utilizable para cualquier género radiofónico, incluido su uso en el 
ámbito educativo mediante narraciones, dramatizaciones y musicales. Permite para su producción el uso de efectos, locuciones y música (Jaimes, 2014). Es un formato histórico dentro del mundo de la radio que ha sobrevivido a los tiempos, sabiendo adaptarse y reformulándose continuamente.

El podcast educativo es audio y video distribuido en archivos digitales de descarga inmediata (Riaño, 2020). Como lo indica Gutiérrez Porlán y Rodríguez Cifuentes (2010), a diferencia de un programa de radio, estos pueden escucharse en cualquier lugar y hora, pues no depende de las programaciones de las radioemisoras. Sin ser para el uso exclusivo de la radio, funcionan como contenido. El cuándo, cómo y dónde oír resultan de una decisión del receptor y no del emisor. Pueden alojarse y/o publicarse en directorios mediante un feed RSS como iTunes, Google Podcasts Spotify, entre otros, así como en redes sociales, Blogs y plataformas educativas como Moodle, Classroom o Edmodo. No necesariamente son de formato breve, pero para uso educativo es pertinente limitar su duración.

Ambos formatos permiten que la información llegue de manera clara y precisa al alumnado siempre y cuando los guiones y el trabajo de pre y post producción sea coherente desde una perspectiva pedagógica, pues como señala Camacho y Castañeda (2010), uno y otro ofrecen frescura y originalidad al proceso de enseñanza y aprendizaje.

\section{FREDER - Fundación Misiones de la Costa - Radio La Voz de la Costa}

La Fundación Radio Escuela para el Desarrollo Rural (FREDER) es una institución creada en la provincia de Osorno, por los hermanos holandeses de la orden capuchina en los años 60 para desarrollar el trabajo misionero en las zonas rurales de San Juan de la Costa. Ellos han generado una política de compromiso social y educativo con las comunidades rurales, campesinas y mapuche huilliche circunscritas al territorio. Desde ella se han generado obras como lo son: la Fundación Misiones de la Costa, administradora de los colegios Qulacahuín y Misión San Juan, los cuales atendieron en el año 2020 a 370 estudiantes de niveles parvulario, básico y medio. La mayoría provenientes de familias campesinas o vinculadas a la cultura mapuche huilliche que habitan en las zonas rurales de la costa de Osorno, aproximadamente a más de 30 kilómetros del centro urbano de la capital provincial.

FREDER impulsó en los años 70 Radio La Voz de la Costa, una emisora con una tradición vinculada al ámbito educativo en la región de Los Lagos. Inició sus transmisiones el 1o de agosto de 1968. Entre los años 70's y 9o's tuvo su auge, siendo partícipes de la convulsionada vida política nacional chilena, desarrollando proyectos de radio escuelas en la región. Fue responsable de la alfabetización de campesinos sin posibilidades de acceso al sistema educativo, logrando importantes índices de egreso y permitiendo la inserción laboral y social de aquellos que participaron de sus programas de capacitación. Actualmente Radio 
la Voz de la Costa opera como medio de comunicación en Amplitud Modulada y está transitando organizacionalmente a ampliar su gestión a Frecuencia Modulada y aumentar su cobertura y potencia. Su presencia activa en el devenir social y cultural del territorio ha ido reduciéndose hasta casi desaparecer (Quintana y Pérez, 2017), manteniendo una política de austeridad que le permite operar con los recursos mínimos, limitando su acción y participación en el campo educativo. Ofrecen apoyo en la difusión de contenidos pedagógicos a los colegios Quilacahuín y Misión San Juan en situación coyuntural, como la del año 2020 y 2021 a raíz de la pandemia mundial por coronavirus.

\section{Metodología}

Los datos que sustentan este artículo forman parte de una investigación más extensa sobre la radio educativa y su accionar en contextos de interculturalidad, específicamente desde su puesta en práctica con alumnado de la cultura mapuche huilliche. Hemos entrevistado a docentes de las escuelas de Quilacahuin y Misión San Juan, así como directivos de la Fundación Misiones de la Costa y personal de Radio La Voz de la Costa, quienes nos han informado sobre el rol educativo de la radio en tiempos de pandemia.

Hemos centrado nuestro interés y objetivos en acopiar relatos de quienes han vivido el proceso de utilizar la radio como recurso didáctico, dialogando con aquellos que, desde el ámbito educativo y su praxis, han puesto en marcha iniciativas innovadoras que han cambiado el enfoque clásico del quehacer pedagógico en momentos donde la opción de la clase presencial está vetada. La metodología que hemos utilizado está circunscrita a un paradigma de tipo cualitativo buscando descubrir las subjetividades y explicar las interacciones y significados de situaciones de la vida real (Álvarez-Gayou Jurgenson, 2003), desde una perspectiva menos positivista y más apegada a los hechos de la cotidianidad sin perder carácter cientificista (Miles \& Huberman, 1984).

\section{Muestra e Instrumentos}

Siendo nuestro caso específico el de recolectar información de primera mano de docentes, directivos y otros informantes, fue necesario hacer una selección acotada de todos quienes han participado de la iniciativa de crear materiales didácticos radiofónicos y organizar instancias de encuentro virtual para poder construir un discurso en base a las experiencias y así evidenciar el rol y uso de la radio educativa como herramienta didáctica en tiempos de coronavirus. Hemos accedido a material de audio, específicamente a cápsulas producidas por el profesorado y las hemos utilizado como recurso para el análisis argumentativo del estudio. Todo material audiovisual supone una importante contribución pues ayuda a analizar y comprender las dimensiones del mensaje educomunicativo (Kaplún, 1998). También logramos acceder a material escrito como por ejemplo 
a la guía para producción radiofónica creada por la Fundación Misiones de la Costa, documento que resultó oportuno para acoplar la triangulación de la información. Los guiones construidos por los docentes también sirvieron de insumos y formaron parte de la información recolectada.

El estar inmersos dentro del contexto educativo de la Región de Los Lagos y en específico en la provincia de Osorno, nos permitió el vínculo con quienes participan de la iniciativa de utilizar la radio como medio de comunicación educativo. A partir de primeros contactos con la directiva de la Fundación Misiones de la Costa, conseguimos concretar espacios de diálogo con un número acotado de docentes entre hombres y mujeres. De ellos decidimos realizar entrevistas a cinco personas, entre las que destacó profesorado vinculado a las áreas de lenguaje, comunicación y cultura. A partir de ahí vinieron las instancias de realización de las entrevistas semiestructuradas como estrategia de recolección de datos, siendo que es una técnica que permite obtener información de forma oral y personalizada, sobre hechos y sucesos, aspectos subjetivos de las personas y grupos, resaltando creencias, valores, aptitudes en relación con el tema de estudio (Sabariego, Dorio \& Massot, 2019). Según McMillan y Schumscher (2005), es una "conversación ordenada" y dirigida hacia el objetivo de la investigación, donde todo lo que se dialoga redunda positivamente como información y dato de interés al estudio. Para el caso específico del momento en que realizamos las entrevistas, la situación de confinamiento imposibilitó que las mismas fueran hechas personalmente, por lo que tuvimos que generar sesiones remotas mediante Meet, aplicación de Google que se utiliza para realizar videoconferencias. Las entrevistas, con una duración de entre 30 y 45 minutos, fueron grabadas y transcritas para su posterior análisis.

\section{Procedimientos}

Toda la información recogida y registrada a través de las entrevistas, más el material audiovisual y de textos, fueron copilados como un corpus de datos para ser analizados. El análisis de datos constituye una etapa clave en el proceso de cualquier investigación de tipo cualitativa (Sabariego, Dorio \& Massot, 2019), por lo que el proceso implicó una necesaria selección de la información mediante categorías a partir de los objetivos propuestos por el estudio, a saber; los modos en que se ha incorporado la radio educativa en 2020 a los tiempos de pandemia, a partir de la experiencia de docentes de dos instituciones educativas en nexo con Radio La Voz de la Costa en la provincia de Osorno al sur de Chile.

El primer momento supuso la revisión de un cúmulo de material escrito, junto a las transcripciones de las entrevistas realizadas. Se hizo una exploración bibliográfica y de referencias, así como artículos de prensa sobre el contexto mundial de la pandemia y sus implicancias en la educación.

Todo el proceso significó una tarea de detección y "desmenuzamiento" de temas y subtemas, de diferenciación y de vinculación, de asociación y de 
comparación, cotejando la relación teórica con la información recolectada y los objetivos del estudio (Ameigeiras, 2006). Para lograr datos confiables, se plantearon unidades de análisis, estas fueron obtenidas de todo el material en bruto (corpus) que se logró recolectar, ordenar y transcribir según los intereses de esta publicación.

Las unidades de análisis se elaboraron por deducción, en un ejercicio de simplificar y encontrarle sentido a toda la complejidad contenida en las entrevistas y material de datos (Patton, 2002). En estricto rigor, se codificó la información en bruto, lo que supuso leer y releer todos los datos recolectados para descubrir relaciones, y en tal sentido comenzar a interpretar (Soneira, 2006).

La segunda etapa significó categorizar las unidades de análisis. Estas categorías recodifican los datos desde una nueva perspectiva teórica, haciéndolos hablar de los procesos y realidades que se evidenciaron en la recolección de antecedentes y permiten comparar fenómenos de semejantes características (Díaz de Rada, 1996). Codificar supone una manera de indexar o categorizar el texto, los audios y videos, para poder establecer un marco de ideas temáticas que nos permitan realizar el análisis (Gibbs, 2012). Esta codificación es descriptiva y suele ser necesario interceptarlas pues van a resultar el núcleo de las posteriores conclusiones. Las categorías significan la estructura más "fina" de la información y al mismo tiempo descifran los datos obtenidos, convirtiéndose en el elemento fundamental para lograr los resultados de la investigación (Álvarez-Gayou Juurgenson, 2003).

El análisis de la información obtenida a través de estas técnicas se trabajó con un sistema de análisis categorial, con orientación hacia un análisis crítico y descriptivo. El material reducido a través de categorías fue discutido y reflexionado junto con el cúmulo de la información obtenida, considerando igualmente soporte teórico para sustentar los planteamientos (Valles, 1999).

Presentamos en el siguiente cuadro todas las categorías que se desprenden de las unidades de análisis de los datos en bruto obtenidos por las entrevistas y el material de análisis, para posteriormente pasar a describir cada una. 
Figura 1. La muestra

Muestra (Datos en bruto)

Entrevistas, audios de cápsulas radiales y material textual

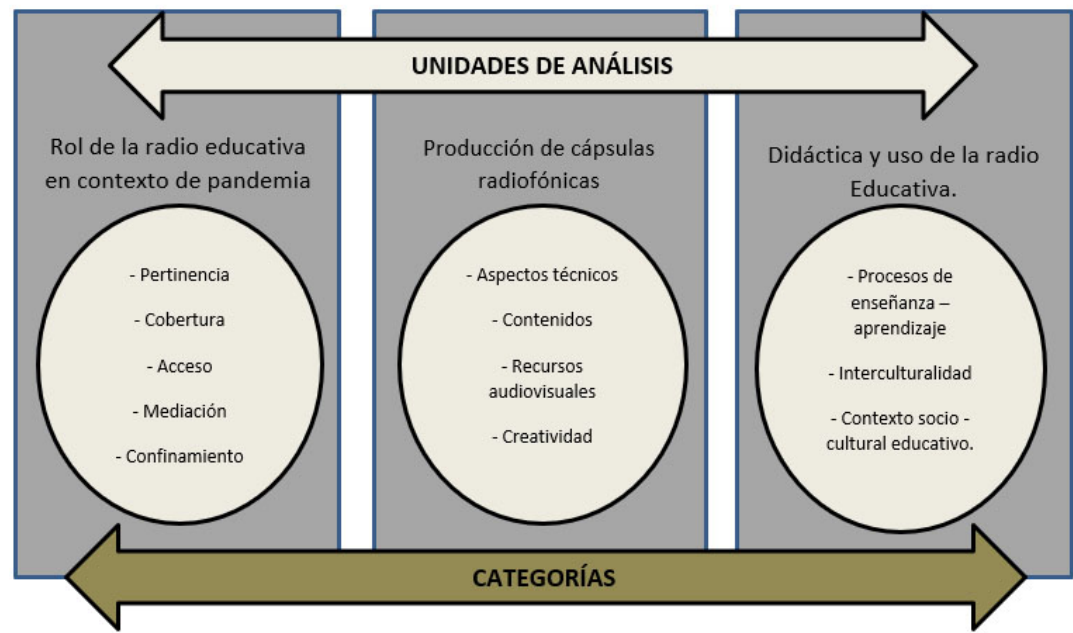

Fuente: Elaboración propia.

\section{Descripción por unidad de análisis y categorías}

\section{Unidad de análisis 1}

1. Rol de la radio educativa en contexto de pandemia.

\section{Categorías}

1.1. Pertinencia: Son los beneficios que la radio puede aportar en momentos donde los sistemas educativos han optado por la no presencialidad, siendo este medio de comunicación una alternativa para la difusión de los contenidos insertos en currículos y planes de estudio. Se pone foco en la particularidad del contexto de la provincia de Osorno.

1.2. Cobertura: Describe cómo la radio educativa puede trabajar con todos los niveles formativos de parvulario, básico y medio, cubriendo aquellas necesidades formativas según las características de cada grupo.

1.3. Acceso: Determina aquellas formas de cómo la radio se vincula con los usuarios, ya que es un medio de comunicación, prestador de servicio público, incluido el ámbito educativo.

1.4. Mediación: Plantea el rol de la radio desde una perspectiva mediadora de los procesos de enseñanza - aprendizaje, donde la relación docente - estudiante 
se ve favorecida por la intervención de este medio de comunicación. Interesa destacar los modos y formas en cómo se produce y llega el mensaje.

1.5. Confinamiento: Sitúa el escenario que ha obligado a las personas al aislamiento en el contexto de la pandemia por coronavirus. Esta situación ha determinado que la educación a distancia y el uso de dispositivos de comunicación para proseguir los planes de estudio sean de uso necesario.

1.6. Brecha tecnológica: Obedece a las diferencias socioeconómicas que existen entre comunidades que tienen acceso a Internet y las que no. Evidencia las desigualdades en el acceso a las TIC, como computadores, Smartphone, Tablet o televisión digital, y cómo ello puede condicionar la continuación formativa de los individuos.

\section{Unidad de análisis 2}

\section{Producción de cápsulas radiofónicas.}

\section{Categorías}

2.1. Aspectos técnicos: Se refiere al uso instrumental de las tecnologías asociadas a la radio, particularmente aquellas referidas a la producción de contenidos: Computadoras, grabadores portátiles de audio, software de edición o micrófonos. Representa aquellos recursos técnicos vinculados al cómo crear contenidos radiofónicos, involucra aquellas destrezas en el manejo de la información a través de redes sociales y todo dispositivo de difusión de materiales audiovisuales.

2.2. Contenidos: Se refieren a las temáticas que abordan las cápsulas radiales, por ejemplo: Historia, lenguaje, matemáticas o cultura mapuche. Los contenidos deben responder a los programas de estudio de los niveles formativos parvulario, básico y medio.

2.3. Recursos audiovisuales: Tratamiento del sonido, la música, los silencios, el lenguaje o la imaginación. Se vincula con la capacidad de crear formatos radiofónicos que transciendan el simple hecho informativo y logren generar interés en los estudiantes radioescuchas.

2.4. Creatividad: Todas aquellas herramientas tecnológicas y del conocimiento que permiten innovar en el proceso de producción de una cápsula radial. Determina situaciones específicas para la resolución de problemas a partir de carencias en habilidades y competencias técnicas. 


\section{Unidad de análisis 3}

\section{Didáctica y uso de la radio educativa.}

\section{Categorías}

3.1 Procesos de enseñanza-aprendizaje: Se refiere a las estrategias utilizadas como mediadoras para hacer efectiva la enseñanza del alumnado mediante el uso de la radio. Establece el uso de metodologías asociadas a las distintas disciplinas de estudio.

3.2 Interculturalidad: Aquello que se relaciona y vincula con el otro. En nuestro caso a la especificidad de la cultura mapuche huilliche y las relaciones, dinámicas y realidades que se generan desde el mundo indígena a partir de las históricas asimetrías generadas por el mundo occidental y sus formas de relacionarse; entre ellas la educación.

3.3 Contexto socio-cultural-educativo: Plantea el entramado de los aspectos y dinámicas sociales tanto de la comunidad de Quilacahuí y San Juan de la Costa, así como las escuelas insertas en ellas, con especial énfasis en lo referido a la interculturalidad representado en lo mapuche huilliche, desde una perspectiva social, cultural y educativa que determina y condiciona comportamientos, formas de relacionarse y cómo se enseña y aprende en esos contextos.

\section{Resultados}

En tanto la unidad de análisis 1; el rol de la radio educativa en contexto de pandemia evidenció que este medio puede ayudar en los procesos de enseñanza y aprendizaje en situaciones coyunturales. La radio logra acortar las brechas tecnológicas que existen en zonas de alta vulnerabilidad y ruralidad, como lo plantea el caso de este estudio. A su vez, la radio sirve a los distintos niveles formativos pues se comprende como un medio de comunicación para el entretenimiento, que enseña mientras se escucha (Peñarrieta Bedoya, 2010).

En cuanto al acceso, se percibe a partir de las entrevistas realizadas, que la radio es entendida como uno de los medios de comunicación más democráticos pues, no condiciona su uso a restricciones económicas, culturales, religiosas o políticas, evitando las grandes brechas ocasionadas por el avance desmedido de las tecnologías y sus limitaciones socioeconómicas (Ballesteros, 2002). Los docentes, al ser consultados sobre la pertinencia de la radio en la coyuntura actual por coronavirus, afirman que ha significado un instrumento de gran valor para dar continuidad a los planes de estudio y acompañamiento afectivo del estudiantado en situación de confinamiento.

Sobre la unidad de análisis 2; producción de cápsulas radiofónicas, las entrevistas y revisión de materiales de audio, así como las cápsulas creadas 
por los docentes, dejó en evidencia que el elemento técnico juega un papel fundamental en el proceso de hacer radio educativa. Deben ser los profesores quienes preparen y produzcan sus propios formatos radiofónicos, adaptándolos a los contenidos programáticos del currículo pues, en muchos casos no existen las capacidades ni competencias técnicas adecuadas para la producción de cápsulas radiofónicas ni podcast, siendo necesario instancias de capacitación para resolver las dificultades en la construcción de los formatos de radio, lo cual implicará también tomar en cuenta espacios para potenciar y desarrollar la creatividad, elemento relacionado a los procesos de innovación educativa.

La Fundación Misiones de la Costa generó un manual que permite completar el proceso de producción de cápsulas, lo cual sirvió de guía y brindó herramientas básicas para, de forma "artesanal", lograr el objetivo de producir. La mayoría de los docentes declaró tener que pedir ayuda de personas más jóvenes, como sobrinos, nietos en incluso estudiantes para poder generar el material didáctico ya que carecían de esas destrezas.

Finalmente, la unidad de análisis 3; didáctica y uso de la radio educativa, demostró que deben resguardarse elementos instruccionales que no solo respondan a los formalismos pedagógicos, sino también a la mediación social que logra la radio como herramienta tecnológica, es decir, su rol en la promoción de valores para la vida y la sociedad (Chirinos, 2014). Si bien el contenido central de las propuestas giraba en torno a los planes de estudio nacionales (lenguaje, cultura, matemáticas, comunicación o ciencias sociales), quedaron demostradas iniciativas que transversalizan los planes formativos, fortaleciendo, por ejemplo, competencias genéricas, como la solidaridad, el buen vivir, el respeto a la diferencia (interculturalidad) y para el caso particular del momento, aquellos temas relacionados al campo de la salud. De esta forma los procesos enseñanza y aprendizaje y las estrategias se vieron nutridos por una perspectiva más humanista que no solo puso el foco en la especificidad técnica, sino también en las destrezas actitudinales. Las estrategias de aprendizaje pueden ser entendidas como el compendio de procedimientos que se instrumentan y conjugan para lograr algún objetivo o plan de aprendizaje (Mayor, Suegas y González, 1995). En el caso de la radio, una de las estrategias que evidenciamos a partir de la narrativa de los docentes, fueron las de tipo metacognitivas. Estas, según Carretero (2001) son aquellas que se generan a partir del conocimiento que las personas construyen respecto del propio funcionamiento cognitivo. Es, en esencia, reflexionar sobre nuestros propios procesos de pensamiento y guiarlos hacia objetivos de aprendizaje significativo o también el conocimiento que tenemos de todas nuestras operaciones mentales (Tishman, 1997). Las cápsulas radiales invitan justamente a operativizar los propios aprendizajes, pues entregan información que debe ser procesada y puesta en diferentes situaciones por el alumnado a partir de sus aprendizajes previos y claro, los contextos en donde se desenvuelven social y culturalmente. 


\section{Discusión}

El año 2020, y el 2021, ha sido extraordinario y circunstancial para la educación a nivel mundial, donde la emergencia sanitaria ha obligado a generar planes de formación a distancia para poder suplir las instancias de presencialidad. Se ha vuelto al uso de la radio como herramienta pedagógica para hacer llegar los contenidos formativos. Esta situación se prueba en aquellos casos donde los usuarios, estudiantes de parvulario o niveles iniciales mapuches huilliches, acceden a los contenidos desde una perspectiva más lúdica, que involucra la imaginación a través del sonido, los efectos o el silencio, como lo hace la radio.

La radiodifusión permite una perspectiva de apertura interactiva de todos quienes asisten y participan de ella, siendo una vía efectiva para todo tipo de mensajes, incluidos aquellos referidos al ámbito educativo. Siendo así, se replantea la representación de que en la actualidad la educación a distancia debe ser mediada únicamente por computadores, quedando demostrado que un medio "cuasi atávico" como la radio, puede cumplir las funciones de intermediario y mediador de la enseñanza en estos tiempos.

En tanto la especificidad de la experiencia que da pie a este artículo, hubo un interesante trabajo colaborativo entre quienes elaboraron las cápsulas radiofónicas (profesorado de las escuelas pertenecientes a la Fundación Misiones de la Costa) y Radio La Voz de la Costa, quien cumplió una labor de intermediario, acoplando y seleccionando los materiales que enviaban las y los docentes, convirtiéndolos posteriormente en un espacio de 30 minutos, donde aparecen las cápsulas en orden temático. Este programa, llamado "A las orillas del Kutralhue", tiene una frecuencia diaria de lunes a viernes a las 14:30 horas y es de asistencia obligatoria para los estudiantes de las escuelas bajo la tutela de la Fundación. Este apoyo en la transmisión ha sido determinante y ha devuelto a Radio La Voz de la Costa su rol histórico de radio educativa en la provincia de Osorno, siendo importante no solo la asistencia técnica que han brindado al proyecto, sino también en la generación de criterios pedagógicos para una buena recepción de los mensajes. Esto supone "empaquetar" los contenidos con una presentación y una despedida, locuciones y anuncios institucionales para publicitar el espacio dentro de su parrilla de programación, así como la elección y filtro de aquellas cápsulas que cumplan requisitos mínimos de calidad para ser transmitidas.

En otro orden de ideas, es importante igualmente señalar que en el ámbito de las emociones existe una trama desde el uso de la radio educativa que se vincula factores que tienen relación directa con los sentimientos, con las relaciones interpersonales y la comunicación. Si se considera el aprendizaje como un elemento de interacción del sujeto con el medio, no se puede dejar de señalar que la actitud positiva hacia sí mismo, hacia los demás y hacia el mismo medio es en definitiva un factor determinante para el éxito de los procesos de enseñanza (García, Gutiérrezy Condemarín, 1997). Desde esta perspectiva, la experiencia ha 
evidenciado el valor de las relaciones alumno-profesor de manera participativa no jerárquica, haciendo énfasis en elementos tales como: La actitud al trabajo, la motivación, la voluntad y las habilidades sociales del propio estudiante. El desarrollo emocional y afectivo no puede estar desligado de una "red social" de elementos que les permitan a los estudiantes competencias para relacionarse simétricamente con el otro y en ello, la radio jugó un rol importante.

\section{Conclusiones}

Sobre los aspectos culturales que evidencia el estudio, hay que resaltar que la mayoría de los estudiantes que componen los colegios Misión San Juan y Quilacahuin se declaran mapuches huilliches o con al menos alguna afinidad con ese pueblo indígena. En un porcentaje aproximado de al menos un 40\%, los contenidos de las cápsulas apuntan hacia ese tema, resaltando todo lo que tiene que ver con la tradición oral, las costumbres y las cosmovisiones ancestrales, así como mitos, leyendas ehistorias que han pasado degeneración engeneración.Las cápsulas radiales buscan revitalizar, optimizar y hacer más eficaz la expresión oral en lengua chesungun (mapuche huilliche), entrelazando con factores transversales como la cultura, la sociedad o la religión, elevando el aprendizaje como un proceso que se sustenta a partir de los contextos, las situaciones, las realidades, los ambientes y las caracterizaciones de los estudiantes, lo cual revela la importancia sobre la pertinencia del aprendizaje multisituado (Sebastián y Scharager, 2007), aquel donde el docente debe "adecuar" el por qué y el para qué de ciertos contenidos en función de una realidad y su contexto. Esto significa que las estrategias no cumplirán su rol específico sino se conjugan con miradas más totalizantes que comprendan lo multifactorial de las dinámicas humanas. Las prácticas pedagógicas desde la radio educativa ponen de manifiesto que los procesos de enseñanza y aprendizaje son dinámicos y susceptibles de variabilidad. Se modelan según las características de los estudiantes y en consecuencias de los contenidos y objetivos de la materia impartida. A partir de las experiencias aquí descritas, se han generado nuevas propuestas en el contexto de los colegios participantes, para seguir utilizando la radio como insumo pedagógico y didáctico una vez pase el tiempo de pandemia, sobre todo en invierno, momento en que muchos estudiantes no acuden a la escuela por las dificultades climáticas que se exacerban en zonas rurales. Para darle continuidad a esta iniciativa radiofónica y educativa, la Fundación Misiones de la Costa ha pensado en la creación de radios escolares en sus establecimientos, lo cual supone generar proyectos para capacitar al profesorado, no solo desde una perspectiva técnica, sino también ofreciéndoles competencias para un uso adecuado de la radio desde lo estrictamente pedagógico. Por último, es pertinente señalar que este estudio exploratorio tuvo como limitación haber sido elaborado y trabajado en un tiempo acotado y particularmente complejo, dadas las circunstancias del contexto en tiempos de pandemia y los problemas 
de comunicación, ya que muchos de los informantes (docentes de las escuelas), viven en zonas rurales y no pudieron participar ofreciendo sus testimonios, lo que sin lugar a duda hubiese enriquecido mucho más los resultados. Entendemos que lo más apropiado a futuro, sería replicar este estudio en otros contextos y momentos.

\section{Referencias bibliográficas}

Aguaded, I., Pérez Escoda A., Peñalva, S. (2018). Integrados comunicacionales: Competencias, TIC y convergencia digital en la transformación de competencias mediáticas. En Ignacio Aguaded y Luis Miguel Romero Rodríguez (Eds.). Competencias mediáticas en medios digitales emergentes. (pp. 211-229). Salamanca: Comunicación Social Ediciones y Publicaciones.

Álvarez-Gayou Jurgenson, J. L. (2003). Cómo hacer investigación cualitativa. Fundamentos $y$ métodos. México D.F.: Paidós.

Ameigeiras, A. R. (2006). El abordaje etnográfico en la investigación social. En Vasilachis de Gialdino (Coord.). Estrategias de investigación cualitativa (pp. 107-151). Barcelona: Gedisa.

Ballesteros, F. (2002). La Brecha Digital. El riesgo de la exclusión en la Sociedad de la Información. Madrid: Fundación Retevisión.

Camacho, M. \& Castañeda, L. (2010). Enseñando y aprendiendo con podcast: Implicaciones educativas, principios básicos de utilización y aplicaciones didácticas. En I.M. Solano (Ed.), Podcast Educativo. Aplicaciones y orientaciones del M-Learning para la enseñanza (pp. 122-136). Madrid: MAD Editorial.

Carretero, M. (2001). Metacognición y educación. Buenos Aires: Aique.

Cohen, D. y Pereira, M. (2010). Lenguajes de la radio. Córdoba: Brujas.

Chirinos, F. (2014). La radio comunitaria como herramienta para promoción de los valores. Berlín: Editorial Académica Española.

Díaz de Rada, A. (1996). Los primeros de la clase y los últimos románticos. Una etnografía para la crítica de la visión instrumental de la enseñanza. Madrid: Siglo XXI de España.

García, C., Gutiérrez, M.C., y Condemarín, E. (1997). A estudiar se aprende. Metodología de estudio por sesión. Santiago de Chile: Ediciones Universidad Católica de Chile.

Gibbs, G. (2012). El análisis de datos cualitativos en investigación cualitativa. Madrid: Morata.

Gutiérrez Porlán, I. y Rodríguez Cifuentes, M.T. (2010). Haciendo historia del Podcast: referencias sobre su origen y Evolución. En Isabel Solano (Editora). Podcast educativo. Aplicaciones y orientaciones del m-learning para la enseñanza. Sevilla: Editorial MAD Eduforma. https://bit.ly/3qzfJHk

Jaimes, D. (2014). Géneros y formatos radiofónicos. Programa Federal de Radios Socioeducativas. Buenos Aires: Ministerio de Educación de la Nación e Instituto Nacional de Formación Docente. https://bit.ly/2HPNıRl

Jaimes, D. (2020). Radios comunitarias, formación y comunicación popular en la Argentina (2011 - 2015). Bernal: Universidad Nacional de Quilmes.

Kaplún, M. (1998). Una pedagogía de la Comunicación. Madrid: Ediciones de la Torre.

Mayor, J. Suegas, A. y González, J. (1995). Estrategias metacognitivas. Aprender a aprender $y$ aprender a pensar. Madrid: Síntesis.

McLuhan, M. (1996). Comprender los medios de comunicación. Barcelona: Paidós.

McMillan, J. y Schumacher, S. (2005). Investigación educativa. Madrid: Pearson. 
Méndiz Rojas, H. (2004). La radio va a la escuela: articulación entre comunicación y educación. Comunicar, (22), 115-120. https://doi.org/10.3916/C22-2004-17

Miles, M. y Huberman, A. (1984). Qualitative data analysis. Beverly Hills: Sage.

Muñoz, J. (1994). Radio Educativa. Salamanca: Cervantes.

Patton, M.Q. (2002). Qualitative research \& evaluation methods. Thousand Oaks, CA: Sage.

Peñarrieta Bedoya, L.G. (2010). Desarrollo de habilidades comunicativas básicas en niños de 9 a 13 años a través de la radio.COMUNI@CCIÓN: Revista de Investigación en Comunicación y Desarrollo, 1(1), 52-61. https://bit.ly/2Zsqot3

Peppino, A.M. (1999). Radio Educativa, popular y comunitaria en América Latina. México D.F.: Plaza y Valdés Editores.

Quintana, M. y Pérez, C. (2017). Juan Meulén, recogedor de imágenes: una historia de apropiación tecnológica. En Cabello, R. y López, A. (Eds.). Contribuciones al estudio de procesos de apropiación de tecnologías (pp. 179-194). Buenos Aires: Ediciones del Gato Gris.

Riaño, F. (2020). Todo sobre Pódcast 2020. Bogotá: Publicación Independiente.

Sabariego, M., Dorio, I. y Massot, I. (2019). Metodología de la investigación educativa. En Bisquera, R. (coord.). Metodología cualitativa (pp. 276-366). Madrid: Editorial Arco.

Sebastián, C. y Scharager, J. (2007). Diversidad y Educación Superior: algunas reflexiones iniciales. Revista Calidad en la Educación, (26), 19-36. DOI: http://dx.doi.org/10.31619/ caledu.n26.231

Soneira, A. J. (2006). La "Teoría fundamentada en los datos" (Grounded Theory) de Glaser y Strauss. En Vasilachis de Gialdino (Coord.). Estrategias de investigación cualitativa. (pp. 153-173). Barcelona: Gedisa.

Tishman, Sh. (1997). El monitoreo mental. En Un aula para pensar: aprender y enseñar en una cultura del pensamiento. Buenos Aires: Ediciones Aique.

UNESCO (Organización de las Naciones Unidas para la Educación, la Ciencia y la Cultura) (2020). Nuevas publicaciones cubanas para enfrentar efectos de la COVID-19 sobre la educación, Oficina de la UNESCO en La Habana https://bit.ly/38elVyc

Valles, M. S. (1999). Técnicas cualitativas de investigación social. Reflexión metodológica y práctica social. Madrid: Síntesis. 
